\section{Getting started in research: research questions, supervisors and literature reviews}

Australasian Psychiatry 2015, Vol 23(1) 8-11 (C) The Royal Australian and New Zealand College of Psychiatrists 2014 Reprints and permissions: sagepub.co.uk/journalsPermissions.nav DOI: 10.1177/1039856214553315 apy.sagepub.com

(S)AGE

\begin{abstract}
Matthew D Macfarlane Committee for Research, Royal Australian and New Zealand College of Psychiatrists (RANZCP), Melbourne, VIC and; Australia Graduate School of Medicine, University of Wollongong, Wollongong, NSW and; Staff Specialist, Illawarra Shoalhaven Local Health District, Wollongong, NSW, Australia
\end{abstract}

Steve Kisely Committee for Research, RANZCP, Melbourne, VIC and; School of Medicine, University of Queensland, St Lucia, QLD, Australia

Samantha Loi Committee for Research, RANZCP, Melbourne, VIC and; Academic Unit for Psychiatry of Old Age, Saint Vincent's Health, Saint George's Hospital, University of Melbourne, Melbourne, VIC, Australia

Jeffrey C Looi Committee for Research, RANZCP, Melbourne, VIC and; Academic Unit of Psychiatry and Addiction Medicine, Medical School, Australian National University, Garran, ACT and; Mental Health Service ACT Health Directorate, Canberra Hospital, Garran, ACT, Australia

Sally Merry Committee for Research, RANZCP, Wellington, New Zealand and; Faculty of Medical and Health Sciences, Werry Centre for Child and Adolescent Mental Health, University of Auckland, Auckland, New Zealand

Stephen Parker Committee for Research, RANZCP, Melbourne, VIC, Australia

Brian Power Committee for Research, RANZCP, Melbourne, VIC and; School of Psychiatry and Clinical Neurosciences, University of Western Australia, Crawley, WA and; Staff Specialist, South Metropolitan Area Health Service, Perth, WA, Australia

Dan Siskind Committee for Research, RANZCP, Melbourne, VIC and; School of Medicine, University of Queensland, Woolloongabba, QLD and; Staff Specialist, Metro South Addiction and Mental Health Service, Brisbane, QLD, Australia

Geoff Smith Committee for Research, RANZCP, Melbourne, VIC and; School of Psychiatry and Clinical Neurosciences, WA Centre for Mental Health Policy Research, University of Western Australia, Crawley WA, Australia

Stephen Macfarlane Committee for Research, RANZCP, Melbourne, VIC, Australia

\begin{abstract}
Objectives: Research can seem daunting, especially for trainees and early career researchers. This paper focuses on how to formulate and begin a research project such as the RANZCP Scholarly Project.

Methods: We outline an approach to framing a research question, developing theses and hypotheses, choosing a supervisor and conducting a literature review.

Conclusions: Through systematic planning early career researchers and other clinicians can plan and conduct research suitable for the Scholarly Project or other research activity.
\end{abstract}

Keywords: educational material, hypothesis, literature review, planning, research methods, research steps, research project structure, qualitative research, quantitative research, scholarly project

$\mathrm{T}$ The Royal Australian and New Zealand College of Psychiatrists (RANZCP) has a strong research focus. Many of its Fellows and affiliates are researchers as well as clinicians. Research and quality improvement is important in the process of improving clinical practice. The path to Fellowship for trainees now includes the requirement to fulfil a 'Scholarly Project', which brings the RANZCP in line with similar requirements in other medical specialties.

This article, written by the members of RANZCP's Committee for Research (CFR), aims to assist trainees, early career researchers, research supervisors and clini- cians with limited research experience; to help provide a 'hands-on' set of advice regarding the conduct of research within an Australian and New Zealand-based context. In addition to these starting tips, useful references and sources of information will be highlighted for further reading.

\section{Corresponding author:}

Matthew D Macfarlane, Graduate School of Medicine, University of Wollongong, Wollongong, NSW 2522, Australia Email: mmacfarlane1@gmail.com 


\section{Demystifying research}

The prospect of putting together a research project can often appear daunting to the first-time researcher; with study design, ethics approval and statistics being the biggest sources of anxiety. There are a number of ways to learn research techniques before beginning a project. These include:

- The critical appraisal of papers in 'Journal Clubs';

- Reading systematic reviews of research, such as the Cochrane Collaboration Reviews; and

- Studying for the 'Critical Appraisal' section of the written examination, for trainees.

Peer-reviewing papers for a journal is a good introduction to research, which will help improve one's scientific writing. It can be useful to ask colleagues whom regularly review journals, about any opportunities to participate in a prospective journal's peer review process for articles, under their supervision. Alternatively, for those clinicians who are confident in taking the lead on reviewing papers, contacting the editors of journals directly and expressing interest is the best way forward.

\section{Coming up with a research question}

During the course of clinical practice, clinicians will encounter questions that are not answered by a review of the existing scientific literature. Maybe no one has done research in the area, or perhaps research was done in other countries or settings, but none has been done yet that could be locally applicable. This could be the opportunity to consider whether a particular research study might fill this gap in scientific knowledge.

It is important to consider what kind of question is to be answered, because this will affect the type of study that needs to be designed. Some common groups of research questions are included in Table 1 . These types of questions are best answered with quantitative studies, of which there are different types.

On the other hand, consider the following research questions:

- How individuals relate to one another, within a particular system;

- Recording the lived experience of individual people and hopefully understanding it better;

- Exploring in-depth opinions and themes about people, in relation to a topic;

- Information that is difficult to reduce to a quantitative measure (for instance, reasoned opinions of study participants on a topic, with pros and cons).
For these questions, qualitative research methods may be more appropriate. ${ }^{1}$

\section{Quantitative research: thesis and hypotheses}

It is useful to break down the research question into an overarching hypothesis: for instance, the thesis may be that Cannabis use increases the risk of developing schizophrenia. This thesis can potentially be broken down into hypotheses, for those whom use Cannabis:

(1) Increased duration of Cannabis use is associated with increased incidence of schizophrenia;

(2) Increased amounts of Cannabis use are associated with increased incidence of schizophrenia, and arising from the first two;

(3) Higher amounts of Cannabis use over 5 years are associated with the increased incidence of schizophrenia.

The development of testable hypotheses helps frame the study design.

Once the research question and hypothesis are settled, they need to be operationalised. This means rewriting the question into a format that is answerable by a research study. There are several ways of doing this: one way is through the 'Population, Exposure, Control, Outcome and Time' (PECOT) formula, described at the University of Auckland's EPIQ website. ${ }^{2}$ While this is designed more for being able to critically appraise existing studies, it is also a good exercise for producing research questions. Many questions, particularly the ones best answered by quantitative methods, can be fitted to this model. For instance, a question such as, 'Does Cannabis cause schizophrenia?' can be expressed as:

'In an adult population in Australia (Population), does cannabis use (Exposure) compared to no use (Control) lead to an increased rate of development of schizophrenia (Outcome) over a period of 5 years (Time)?'

Framing the question in this way gives some idea of what type of study would be the best way to answer it. It will also assist in focusing on specific details that may otherwise be overlooked. In this case:

'What are the characteristics of the population to be focused on? Should it be limited to a particular age group, relating more closely to everyday practice? How is Cannabis use defined? Is the amount of Cannabis smoked, or the duration of use, important? How should the development of schizophrenia be monitored? Is the timeframe realistic, given the resources of the service or existing data, and is it long enough to answer the question? Are there confounding factors that should be considered?' 
Table 1.

\section{Research question}

How common is a particular condition within a specific population?

How effective is a treatment, compared to another treatment or to placebo?

Is this historical factor associated with the development of an illness?

Is this diagnostic or screening tool accurate? What are its strengths and Diagnostic study

weaknesses?

Is your service following evidence-based guidelines in a given area of practice?

Is a new service programme more effective than usual care?

What does the published literature say about a particular treatment's

effectiveness?

RCT: randomised controlled trial

\section{Study design}

Observational/epidemiological study

Clinical trial/RCT

Case-control or cohort study

Quality improvement/clinical audit

Health services research/quasi-experimental designs Systematic review or meta-analysis

\section{Qualitative research: defining questions and sampling}

In contrast to quantitative research, in qualitative research the aim is to collect information the leads to a greater understanding of a problem. ${ }^{3}$ It may be used to generate hypotheses that can then be tested in a quantitative study. The sample is usually small and is not required to be representative. Data collection is unstructured or semi-structured, as there is an attempt to encourage people to express their views independently of the researcher. Data analysis is not statistical, but involves an examination of the responses of the participants; which can be very detailed, as in the case of discourse analysis, or can be broad and 'thematic'. A popular approach in the medical field is to use general inductive analysis. ${ }^{4}$ The findings are exploratory and they are used to develop an initial understanding of a problem that can then lead to further investigation. There are specific theories that dictate the design and analysis in qualitative studies and if a qualitative study is being planned, it is prudent to seek advice from someone with skills in this area.

\section{Don't overreach}

It is important to consider the scope of any proposed study before it starts. Modern psychiatric researchers typically work in multi-disciplinary teams. As such, some research questions are beyond the scope of a sole investigator (e.g. randomized clinical trials (RCT) involving new interventions and risk factor epidemiology involving the collection of large samples). Refining the study question, as just discussed, can allow the production of a study design that is specifically focussed on answering it. The end result may be a study that can effectively answer a far more modest question than the question originally posed; yet this is generally better than having a study that fails to answer any question, due to over-ambition.

Sometimes data have already been collected in the area of interest and may be available to a junior researcher for analysis; in this instance, the original research question may have to be reframed because of limitations in the existing data. Just because the study question may have changed does not make the project a failure: on the contrary, it is an important part of learning about research.

\section{Choosing a supervisor}

Once the research question has been settled, the next step is finding a supervisor. Ask local senior colleagues, university departments or the Director of Training for advice. Bear in mind that a supervisor may be a practicing clinician or can be someone without a direct clinical load. Depending on the type of research, a career researcher might be a better option than someone with a busy clinical practice, as linking up with established research teams (if one is working in the area) can be very useful for acquiring particular research skills. The nature of these tasks will need to be approved by the local College Trainee Committees, but could include tasks such as:

- Complete formal training on research methodology;

- Learn how to use diagnostic or clinical instruments;

- Learn how to conduct statistical analyses;

- Learn how to do certain types of data analyses, such as qualitative thematic analysis; 
- Write up a full research protocol and the associated patient information sheet and patient consent forms.

Thus, one can complete 'real life' components of a full, modern research experience, but have realistic goals set in collaboration with experienced researchers. Trainees should bear in mind that if the primary supervisor is a non-RANZCP Fellow researcher, the Scholarly Project guidelines also require a local RANZCP Fellow to co-supervise.

Meet with the potential supervisor, to see if there are mutual interests in research and to gauge your compatibility of working styles. Inexperienced researchers need to ensure that any potential supervisor is willing to help through some of the basics, such as looking over and correcting research proposals and ethics applications; helping with basic questions surrounding methodology, including data collection and analysis (statistical methods); and giving feedback on the study write-up. Some supervisors are interested in the end product, the published paper, but aren't very hands-on when it comes to the process of producing it. Putting together a written supervision agreement is often a good idea, to clarify the supervisory relationship.

Speaking to previous students of the supervisor, particularly those who have finished their research and moved elsewhere, can be useful: they are more likely to give an honest opinion.

\section{The literature review}

While the study topic may be novel, almost all research builds on the knowledge of others in the field. It is important to be abreast of historical and current developments in the topic of the research project. In order to do this, every study should be preceded by a review of the scientific literature. This will give an understanding of the previous work, as well as potential opportunities and pitfalls in relation to the topic.

In addition to searching electronic/online databases (such as Medline, PsycINFO, Embase and CINAHL) for keywords related to the topic, it is useful to comb the reference section of each article located, to see if there are other relevant articles in the field. If an article is cited frequently, it is likely to be a good reference to seek out. Review articles in the area may have done some of the literature-reviewing work; if so, read them thoroughly and explore their reference lists. A literature review will assist in refining the research question, and making it more likely to be novel and useful.

The time of the literature review is also the time to begin collecting the references for any future write-up. Consider using an electronic reference manager, such as Endnote ${ }^{5}$ or Refman, ${ }^{6}$ if there are a large number of citations. Choose one that can be linked to common computer word processing programs.

\section{Next steps}

Having framed the research project, the next step is to move on to the design of the study, with support from your supervisor and collaborators. The RANZCP website provides a useful reference for early career researchers (see www.ranzcp.org/Publications/Research/ Research-in-Psychiatry.aspx).

\section{Disclosure}

The authors report no conflict of interest. The authors alone are responsible for the content and writing of the paper.

\section{References}

1. Kitto SC, Chesters J and Grbich C. Quality in qualitative research. Med J Austral 2008; 188: 243-246.

2. Effective Practice, Informatics \& Quality Improvement (EPIO). University of Auckland. Evidence-based practice and critical appraisal, www.fmhs.auckland.ac.nz/soph/depts/ epi/epiq/ebp.aspx (2014, accessed 12 April 2014).

3. Fossey $E_{\text {, Harvey } C, M c D e r m o t t ~}$, et al. Understanding and evaluating qualitative research. Austral NZ J Psychiatry 2002; 36: 717-732.

4. Thomas DR. A general inductive approach for analyzing qualitative evaluation data. American Journal of Evaluation 2006; 27: 237-246.

5. Endnote X7. Thomson Reuters, www.endnote.com (accessed 14 July 2014).

6. RefMan 12.0.3. Thomson Reuters, www.refman.com ( accessed 14 July 2014). 